\title{
Parque fluvial en quebrada San Francisco, Valparaíso
}

CAMILA BIDART SANTANDER

\author{
$>$ Arquitecta, Fundación Cuencas de Chile \\ www.arquitecturabidart.cl | cuencaschile.org \\ ORCID 0000-0003-4266-2136
}

Universidad de Valparaíso Facultad de Arquitectura Revista Márgenes Espacio Arte Sociedad Parque fluvial en quebrada San Francisco, Valparaíso

Octubre 2021 Vol. $14 \mathrm{~N}^{\circ} 20$

Páginas 33 a 41

ISSN elec. 0719-4463

Recepción: Diciembre 2020

Aceptación: Mayo 2021

doi.org/10.22370/margenes.2021.14.20.2997

\section{RESUMEN}

La ciudad de Valparaíso presenta una serie de cuencas hidrográficas que emergen a partir de las altas pendientes de sus cerros en dirección al Océano Pacífico, y cuya morfología es capaz de brindar servicios eco-sistémicos. En particular, el sistema de cuencas generado a partir de la quebrada San Francisco resulta de especial interés, ya que actúa como un corredor biológico, teniendo así el potencial de preservar la biodiversidad local. Considerando estos atributos, y a raíz del descubrimiento de un sistema de recolección de aguas lluvias abandonado que tenía como fin encauzar las aguas de éste y de otros sistemas hidrográficos de la ciudad, se propone la construcción de un parque fluvial que enlaza la naciente de la cuenca San Francisco con su desembocadura en el barrio puerto. Este proyecto busca por un lado poner en valor el sistema de cuencas hidrográficas de la ciudad, y dar cuenta de sus propiedades ecológicas. Así también, se desea potenciar este hallazgo arqueológico, parte del patrimonio histórico de la ciudad, acogiendo el flujo natural de agua que dio origen a su construcción.

\section{PALABRAS CLAVE}

cuencas hidrográficas, servicios eco-sistémicos, corredor biológico, patrimonio natural, patrimonio arqueológico, parque fluvial

\section{River park in San Francisco Ravine, Valparaíso}

\section{ABSTRACT}

The city of Valparaíso has a series of watersheds that emerge from the high slopes of its hills into the Pacific Ocean, and whose morphology is capable of providing ecosystem services. In particular, the watershed system generated from the San Francisco ravine is of special interest, since it acts as a biological corridor, thus having the potential to preserve local biodiversity. Considering these attributes, and as a result of the discovery of an abandoned rainwater collection system which purpose was to channel the waters of this and other hydrographic systems of the city, it is proposed the construction of a river park that links the source of the basin San Francisco with its mouth in the port area of the city. This project seeks, on the one hand, to value the city's hydrographic basin system, and to account for its ecological properties. Likewise, it is desired to enhance this archaeological find, part of the historical heritage of the city, welcoming the natural flow of water that gave rise to its construction.

\section{KEYWORDS}

watersheds, ecosystem services, biological corridor, natural heritage, archaeological heritage, river park 


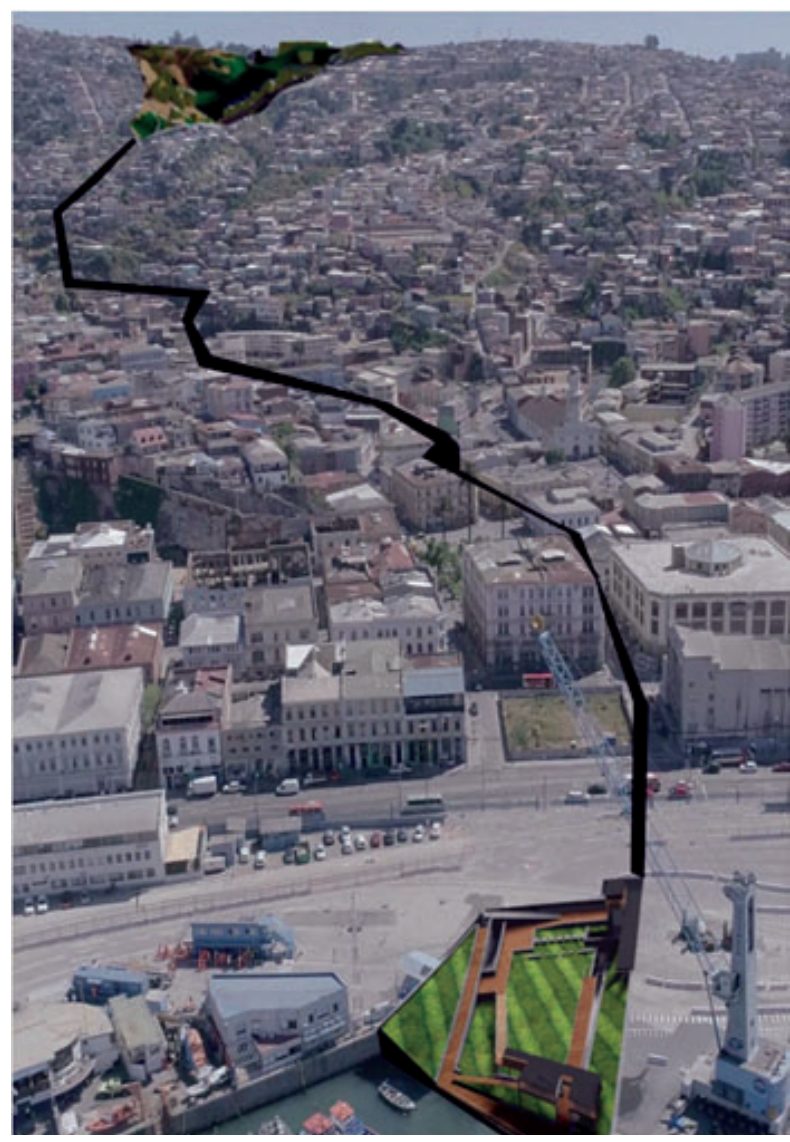

\section{INTRODUCCIÓN}

La ciudad de Valparaíso presenta una serie de cuencas hidrográficas que emergen a partir de las altas pendientes de sus cerros en dirección al Océano Pacífico, comprendiendo una cuenca hidrográfica de la misma manera con que se entiende el concepto de cuenca colectora o de captación, siendo ésta toda la porción de superficie terrestre cuyas aguas vierten a un modelo orgánico que se arraiga a la geografía (Martínez Duarte, 2006:2).

Así, desde la zona portuaria de la ciudad se distingue un sistema de quebradas que gracias a su morfología es capaz de brindar servicios eco sistémicos, siendo estos los componentes y procesos de los ecosistemas que son consumidos, disfrutados o que conducen a aumentar el bienestar humano tomando en cuenta la demanda de los beneficiarios, así como la dinámica de los ecosistemas (Daily, 1997:3). Estos servicios resultan por ende esenciales para el habitar sostenible del territorio, abarcando no solo la mantención y producción de agua, sino también la formación de suelo, la regulación del clima por parte de los bosques, y servicios culturales como la recreación y contemplación de la naturaleza, entre otros aspectos.

Junto con esto, el sistema de cuencas hidrográficas de Valparaíso, y en particular el sistema generado por el cauce San Francisco que conecta con el sector de Laguna Verde por el sur oeste, actúa como un corredor biológico ya que permite enlazar dos o más sectores con características ambientales similares, de forma que resulta transitable y sirve como lugar de desplazamientos para las especies de la zona (Gurrutxaga, Lozano, 2008:173). Esto contribuye a preservar la biodiversidad de la zona, prevenir la fragmentación de los hábitats costeros, y fomentar la migración, dispersión, vinculación e interrelación de especies de flora y fauna nativa, todos elementos indispensables para la conservación del patrimonio natural de la región.

Considerando todas estas cualidades, y a raíz del hallazgo en 2015 de un sistema de evacuación de aguas lluvias construido a mediados del siglo XIX, el equipo de Cuencas Chile liderado por la arquitecta Camila Bidart, realizó en septiembre de 2017 el estudio y diseño de un parque fluvial en la quebrada costera de San Francisco, en la comuna de Valparaíso. Este proyecto busca por un lado poner en valor el sistema de cuencas hidrográficas de la ciudad, entendiendo que es una matriz orgánica capaz de encauzar y filtrar las aguas que confluyen en la bóveda encontrada. Así también, el diseño del parque contempla potenciar este hallazgo arqueológico, parte del patrimonio histórico de la ciudad, acogiendo el flujo natural de agua que dio origen a su construcción en la zona típica de Valparaíso.

\section{ANTECEDENTES DEL PROYECTO}

\section{Morfología y Cuencas Hidrográficas de Valparaíso}

La morfología de Valparaíso se caracteriza por ser una ciudad construida sobre un sistema de cuencas hidrográficas proveniente de sus cerros y orientadas hacia el océano pacífico, vinculando transversalmente la zona del puerto con un sistema de quebradas en que se transporta el agua a través de su pendiente, generando redes hídricas de agua dulce.

Para el caso del cauce San Francisco, en la cabecera de su cuenca se distingue la línea divisoria de aguas Iluvias. Siguiendo el curso de las aguas pluviales, se encuentra el fondo de la quebrada, un 
espacio entre laderas de altas pendientes, que dada la morfología permite la existencia de bosque esclerófilo, el cual es capaz de acumular y filtrar las aguas lluvias que bajan desde su cabecera.

Este modelo orgánico ordena y protege los flujos de agua naturalmente, generando servicios eco-sistémicos como el recién mencionado, que a través de los elementos radiculares de las especies nativas que allí habitan, estratifican el bosque con sus coberturas. En consecuencia, se crea también un sistema de depuración, ya que la concentración de los flujos de agua en los pliegues del cerro junto a la densificación del bosque contribuye a la acumulación de agua en napas subterráneas, generando un mayor drenaje de los escurrimientos y evitando así derrumbes y aluviones (Martínez Duarte, 2006:8).

Además, el cauce forma parte de un eje que atraviesa transversalmente desde el puerto de la ciudad (norte) hacia el sur oeste, generando un flujo que conecta el sector de Laguna Verde con el barrio puerto mediante un corredor biológico, proporcionando conectividad entre paisajes, ecosistemas y hábitat, y asegurando el mantenimiento de la diversidad biológica y los procesos ecológicos y evolutivos (García, 1996:44)

En relación a la espacialidad de la cuenca, ésta comprende múltiples orientaciones enfrentadas que conforman las laderas, las que permiten el desarrollo de un microclima húmedo que acoge a la flora nativa. Este microclima se divide por el cauce en dos zonas, una ladera orientada hacia el norte, y otra hacia el sur.

La ladera solana (norte) se encuentra erosionada debido a los asentamientos humanos y el aterrazamiento de la pendiente, mientras que la ladera umbría (sur) tiene mayor vegetación gracias a sus fuertes pendientes por donde escurre el agua, manteniéndose la humedad. De esta forma, la condición geográfica de una cuenca hidrográfica influye tanto en su capacidad de permitir asentamientos como de brindar servicios eco-sistémicos como los antes mencionados.

\section{Convivencia entre factores Antrópicos y Naturales}

El poblamiento humano con su inherente alteración del entorno sin duda puede llegar a afectar la capacidad que tienen los modelos orgánicos antes mencionados para brindar servicios eco-sistémicos, siendo un problema que, si bien siempre ha existido, recién hoy en día se comienza a estudiar con mayor profundidad. Esto es de suma relevancia para el caso de Valparaíso, ciudad que históricamente ha crecido por medio de una urbanización no planificada que convive con los elementos naturales del paisaje, donde la morfología de sus quebradas y sus pliegues construidos por senderos y cauces de agua traman conectividades, centros e intercambios, que dan origen a los espacios públicos.

Con respecto al sistema de cuencas hidrográficas de la ciudad, el hallazgo arqueológico de un sistema de evacuación de aguas lluvias del siglo XIX durante la ejecución del proyecto Mejoramiento de Espacio Público del Barrio Puerto, realizado por la constructora PADECASA en el año 2015 y que consistió en la reposición del adoquín de las calles Clave y San Martín, otorga ciertas pistas y herramientas clave para conocer y comprender tanto el pasado como el estado actual de la convivencia entre el modelo orgánico de flujo de aguas con el entorno hoy altamente urbanizado.
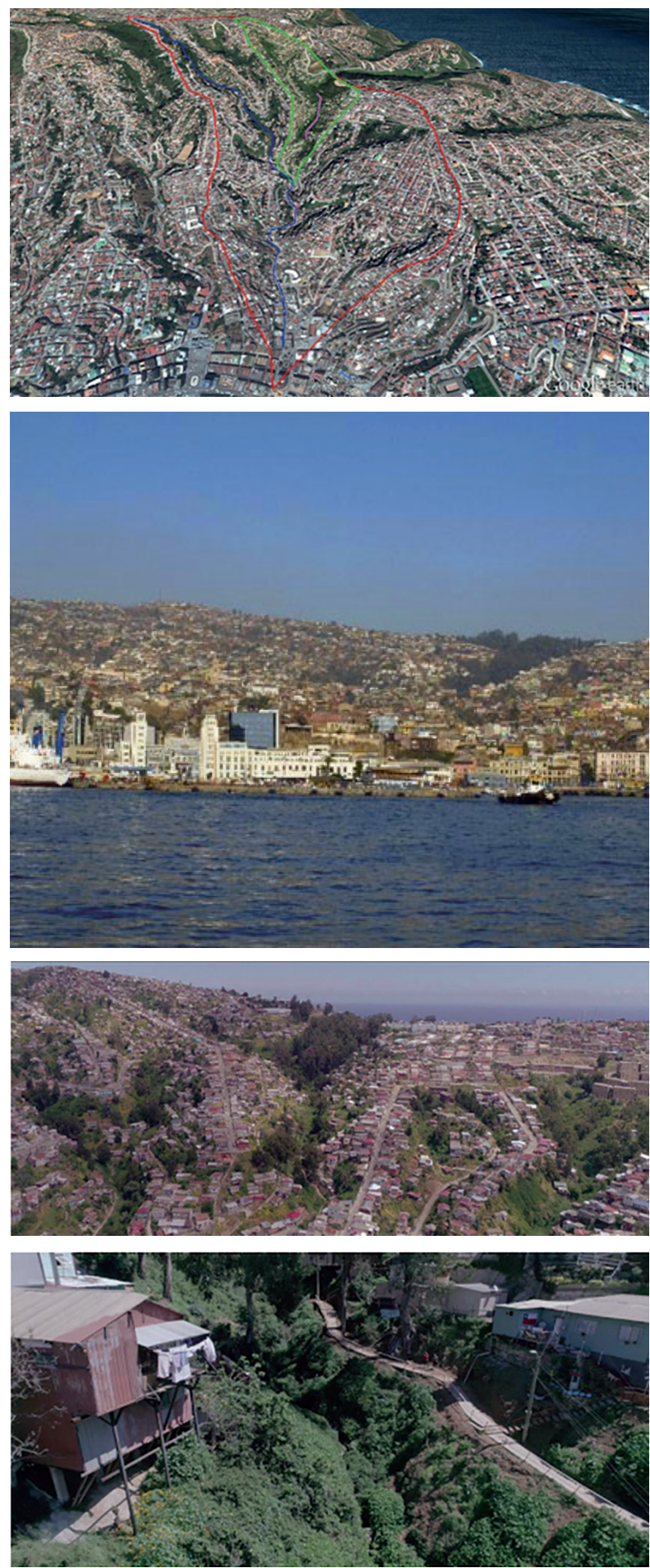

$>$ Figura 2. Área que abarca la Cuenca San Francisco. Fuente: Elaboración del autor.

$>$ Figura 3. Bosque esclerófilo de la quebrada San Francisco visto desde el mar. Fuente: Tripadvisor.

$>$ Figura 4. Sistemas de quebradas que conforman la cuenca San Francisco. Fuente: Registro del autor.

Figura 5. Asentamientos en la quebrada San Francisco. Fuente: Registro del autor. 

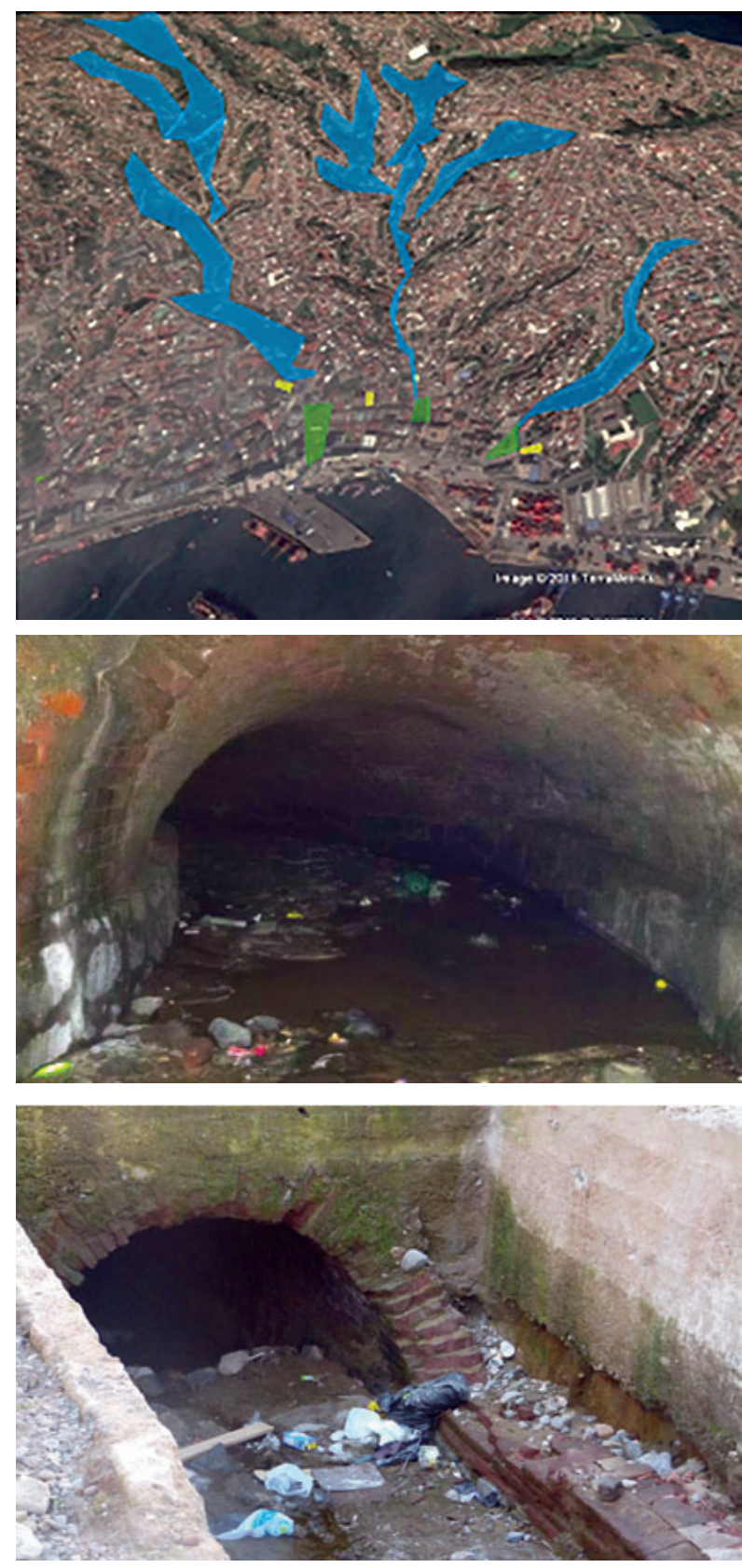

$>$ Figura 6. Cauces de agua y su conexión con los espacios públicos de la zona típica. Fuente: Elaboración del autor.

> Figura 7. Bóvedas de agua al momento de ser descubiertas, 2015.

$>$ Figura 8. Bóvedas de agua al momento de ser descubiertas, 2015.

\section{Aproximación histórica}

Durante el siglo XIX la ciudad vivía una etapa de plena expansión urbana y de aumento poblacional. Bajo este contexto, surge la necesidad de urbanizar la ciudad desde su borde, obstaculizando el curso natural de las aguas provenientes de las cimas que desembocaban al mar (Muga, Rivas, 2009:207). El estancamiento de estas aguas derivó en problemas de insalubridad de los espacios públicos y naturales, generando un escenario propicio para la proliferación de bacterias y enfermedades, siendo una de las más masivas una epidemia de Escarlatina ocurrida entre los años 1831 y 1832 (Urrutia, Lanza, 1993:86), antecedente que propicia la instauración del discurso higienista europeo. Así, tras una búsqueda del origen de las plagas se llega a la conclusión que existía una relación entre las aguas estancadas y la emanación de vapores en pantanos Ilamados “miasmas" (Urteaga, 1980:7), siendo éstas una emanación nociva que se suponía desprendían los cuerpos enfermos o materias en descomposición.

A su vez, la urgencia del recurso hídrico para sobrevivir generó en la sociedad la necesidad de organizarse y diseñar redes de colectividades, así como también el entendimiento del territorio que se habitaba en relación al agua. Surge entonces en 1830 la "Policía Urbana", con el fin de materializar obras de saneamiento y comenzando con la construcción de redes de agua y alcantarillado, pavimentación de calles y control de las quebradas mediante evacuaciones subterráneas de los cauces naturales como medio de llegada al mar (Alvares, 2001:6).

Esta nueva forma de entender la ciudad, enfocada en la hidrología urbana, genera una idea más integrada de los procesos de drenaje de las aguas lluvias, comprendiendo que un manejo adecuado de los cauces naturales que bajan desde la cima hacia el mar era necesario tanto para no interrumpir la urbanización de Valparaíso en relación al Puerto, como para proteger a la comunidad de enfermedades producidas por la contaminación y mal manejo de las aguas.

\section{Sistema de evacuación de aguas lluvias para Valparaíso}

Es entonces que, mediante la tecnología como instrumento técnico y los aspectos higiénicos como fundamento, se da lugar a las obras de construcción del sistema de evacuación de aguas lluvias descubierto en los últimos años, el cual comenzó a implementarse a partir de 1850 por el ingeniero Guillermo Wheelwright. Los trabajos comenzaron en la quebrada San Agustín, construyendo allí una captación y un estanque, y se tendieron cañerías de fierro fundido por La Planchada (actual calle Serrano), y por San Juan de Dios (Condell) hasta la calle del circo (Edwards) (Alvares, 2001:9). De esta forma se empezó a encauzar los flujos de agua más importantes de la ciudad, entre ellos el Cauce San Francisco, conocido por su gran caudal. Se creó así una forma de "excreción subterránea", siendo el borde mar el límite natural donde convergen las aguas de las cuencas con las aguas negras producto de los asentamientos humanos, todas encauzadas bajo el casco histórico de la ciudad.

Esta ubicación no vendría a ser una casualidad, ya que como se puede observar en la imagen, existe una directa relación y conexión entre el origen de los espacios públicos de la zona típica (en verde) con los cauces más importantes que bajan desde la cima y que convergen en el plan (en azul), entendiéndose así que el relleno y la urbanización dio origen a las plazas Aduana del cauce Carampagne, Plaza Echaurren del cauce San Francisco y la plaza Soto Mayor, donde desemboca el cauce Tomas Ramos. 


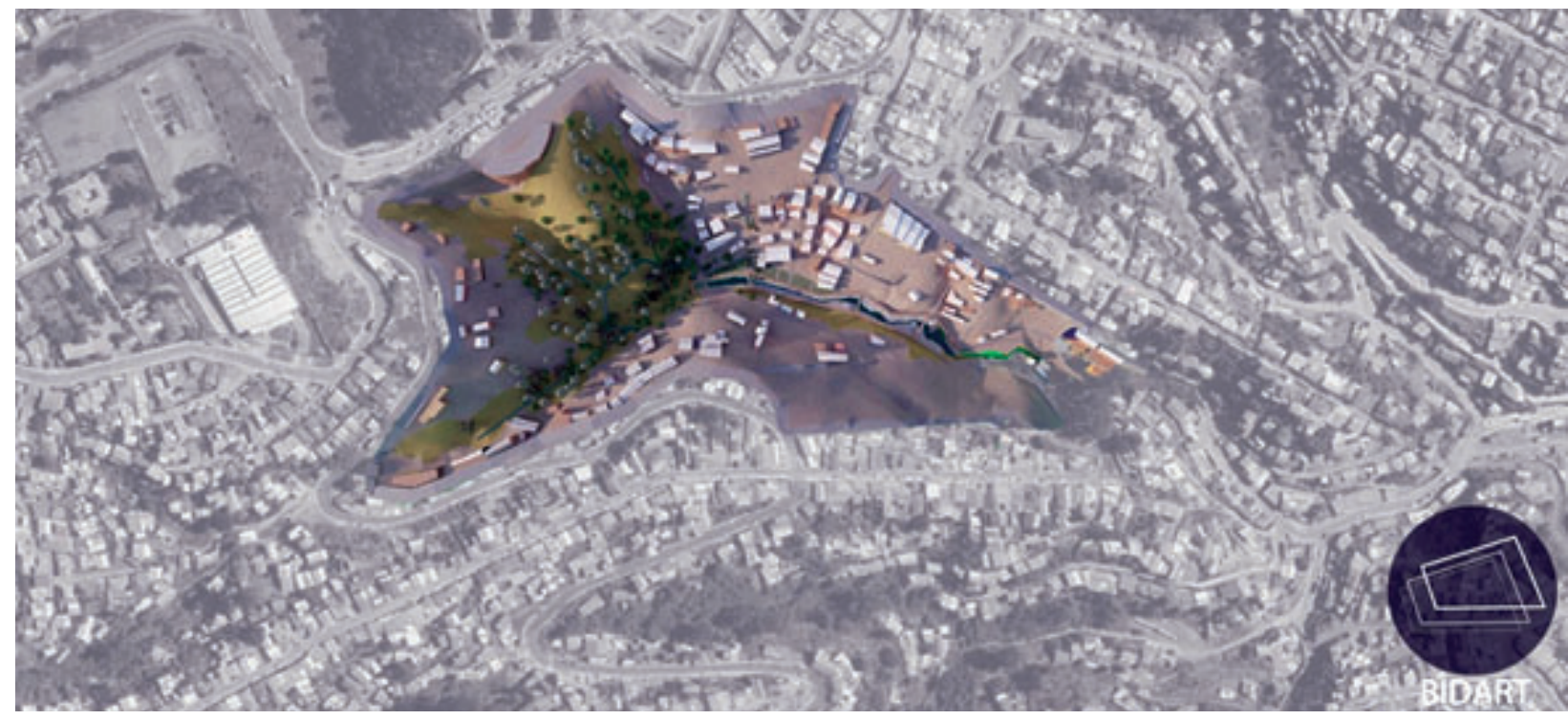

En referencia a la obra descubierta, ésta es una bóveda de $3 \mathrm{~m}$ de altura por $3 \mathrm{~m}$ de ancho que recorre subterráneamente el Barrio Puerto, y que está compuesta por losas y soluciones mixtas de pretiles y arcos que cubren así los cauces en su totalidad hasta llegar al mar.

De esta forma, y comprendiendo la morfología de los espacios en relación al agua, el cauce San Francisco abovedado es parte de un sistema que recorre transversalmente en forma de una gran avenida, con un ancho pronunciado y articulado mediante nodos y puntos de inflexión hasta llegar a la plaza Echaurren, en donde desemboca subterráneamente en el mar mediante un cauce. Así, se ha podido evidenciar que la evacuación de aguas desde la quebrada San Francisco ha sido abordada desde el siglo XIX hasta la fecha mediante un colector que nace en el empalme del Camino la Cintura con la Avenida Alemania, pasando por el subsuelo de la ciudad hasta desembocar en la bahía, en el que actualmente se realiza un dragado permanente del puerto para solucionar el problema de embancamiento de arena.

\section{PARQUE FLUVIAL EN QUEBRADA SAN FRANCISCO}

La Quebrada San Francisco, con su enorme atractivo patrimonial y sociocultural, posee los atributos para formular un proyecto de uso del borde costero interconectado con la biodiversidad ambiental y cultural de la naciente de la quebrada.

Por un lado, el proyecto nace a raíz del descubrimiento de la bóveda en el barrio Puerto, teniendo como objetivo preservar, conservar y mantener vigente una construcción que data del siglo XIX, siendo una muestra tangible del desarrollo cultural y la ingeniería hidráulica urbana de esos tiempos.

Asimismo, se tiene el objetivo de restaurar el ciclo del agua para el ecosistema formado en esta quebrada costera, y al entender el origen de la acumulación de ésta, el fondo de la quebrada, permite considerar y potenciar también el corredor biológico existente entre este punto y el plan de la ciudad.

De esta forma, la consolidación de este borde urbano resulta fundamental para la conducción, protección, y mantención del cauce de aguas lluvias desde la cima al borde mar, lo cual es posible por medio de un paisaje que es capaz de mejorar la calidad de vida, salud, y conectividades de las comunidades que habitan el lugar.
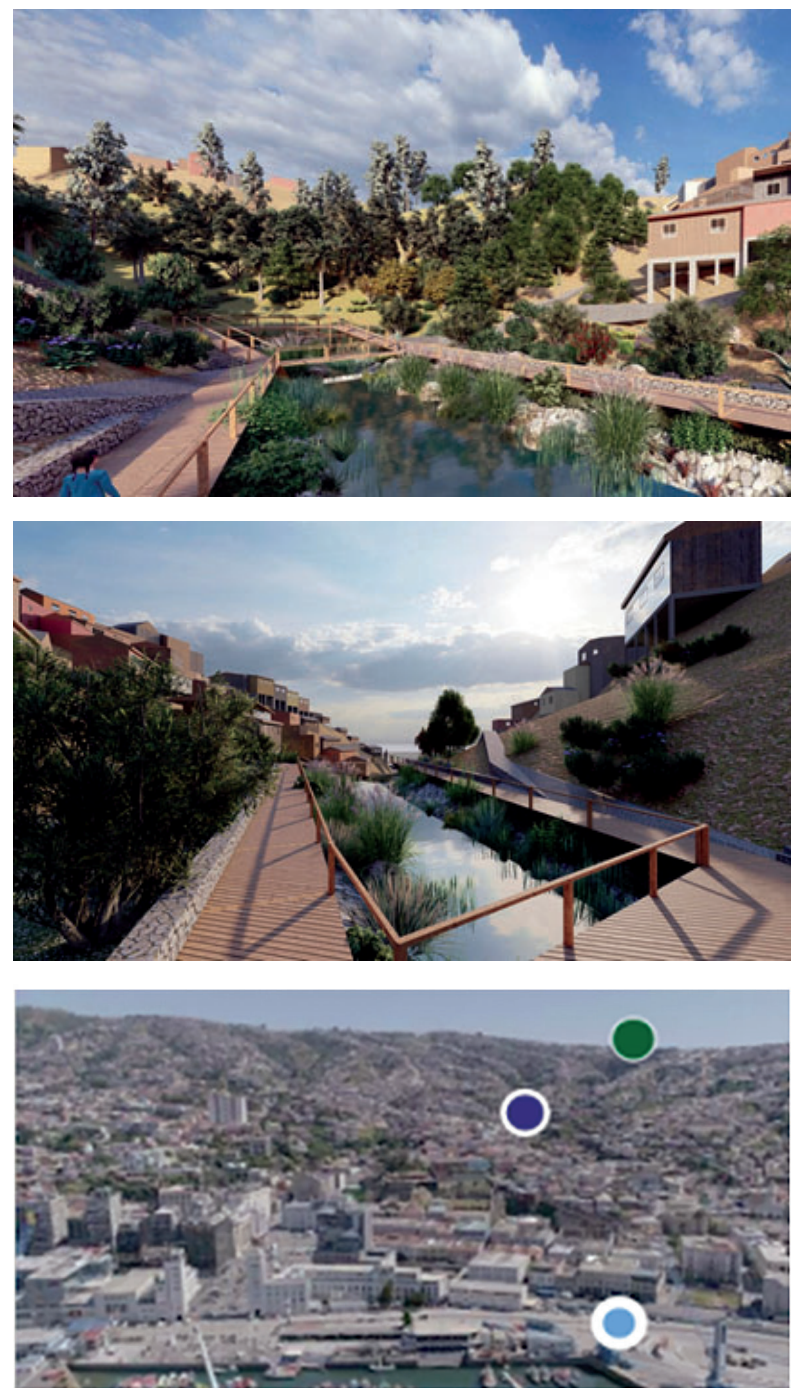

$>$ Figura 9. En color se destaca el área del punto de intervención en la naciente de la quebrada. Fuente: Bidart Arquitectos.

$>$ Figura 10. Maqueta 3D del Parque Fluvial. Fuente: Bidart Arquitectos.

$>$ Figura 11. Maqueta 3D del Parque Fluvial. Fuente: Bidart Arquitectos.

$>$ Figura 12. Principales puntos de Intervención del proyecto. Fuente: Elaboración del autor. 

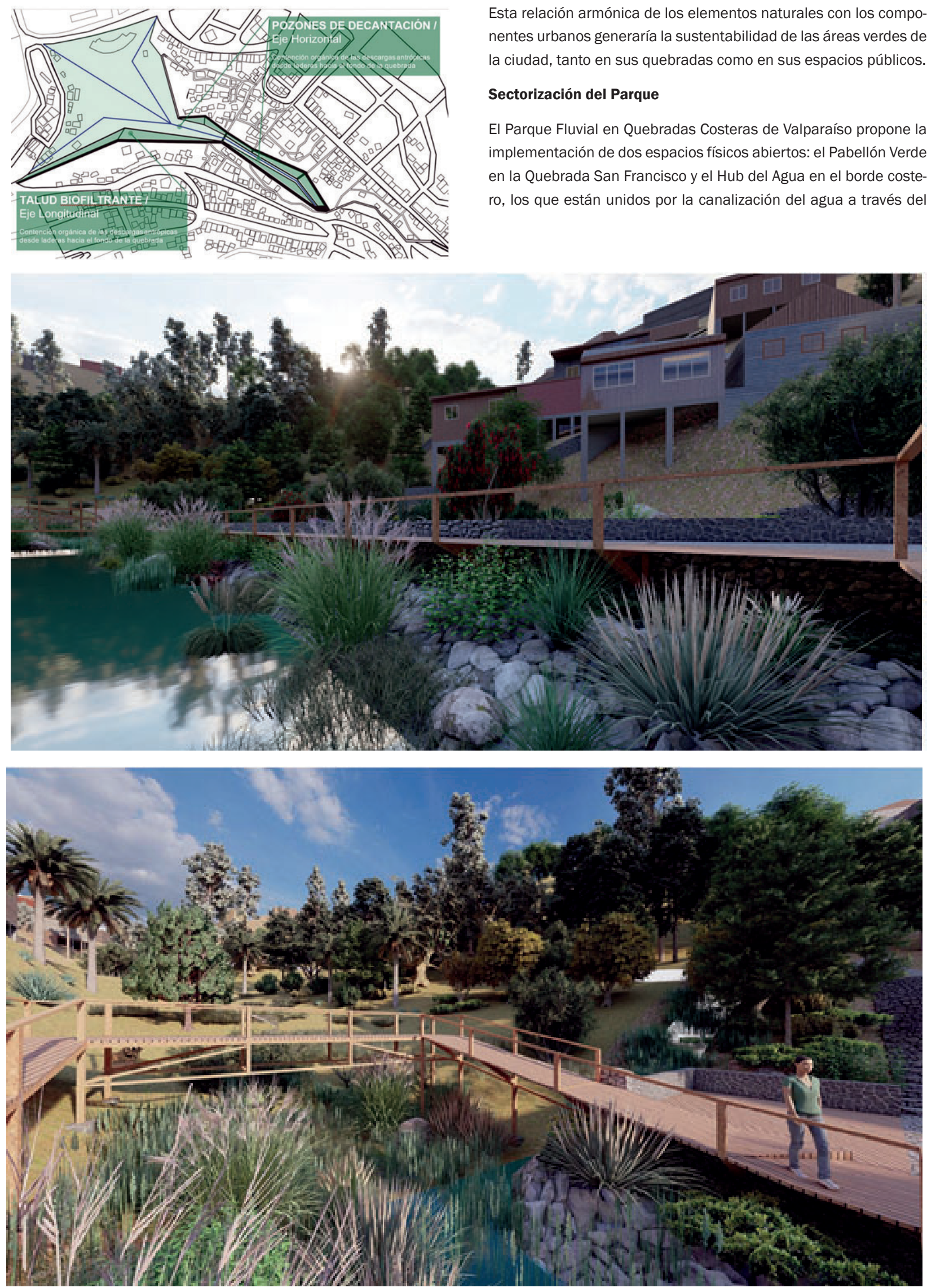

$>$ Figura 13. Plano que destaca en verde la ubicación de los pozones de decantación y talud biofiltrante. Fuente: Bidart Arquitectos.

$>$ Figura 14: Maqueta 3D del Parque Fluvial. Fuente: Bidart Arquitectos.

> Figura 15. Maqueta 3D del Parque Fluvial. Fuente: Bidart Arquitectos.

38 > Revista Márgenes N²0 Vol 14 > Octubre 2021 páginas 33 a 41
Esta relación armónica de los elementos naturales con los compola ciudad, tanto en sus quebradas como en sus espacios públicos.

\section{Sectorización del Parque}

El Parque Fluvial en Quebradas Costeras de Valparaíso propone la implementación de dos espacios físicos abiertos: el Pabellón Verde en la Quebrada San Francisco y el Hub del Agua en el borde costero, los que están unidos por la canalización del agua a través del nentes urbanos generaría la sustentabilidad de las áreas verdes de 

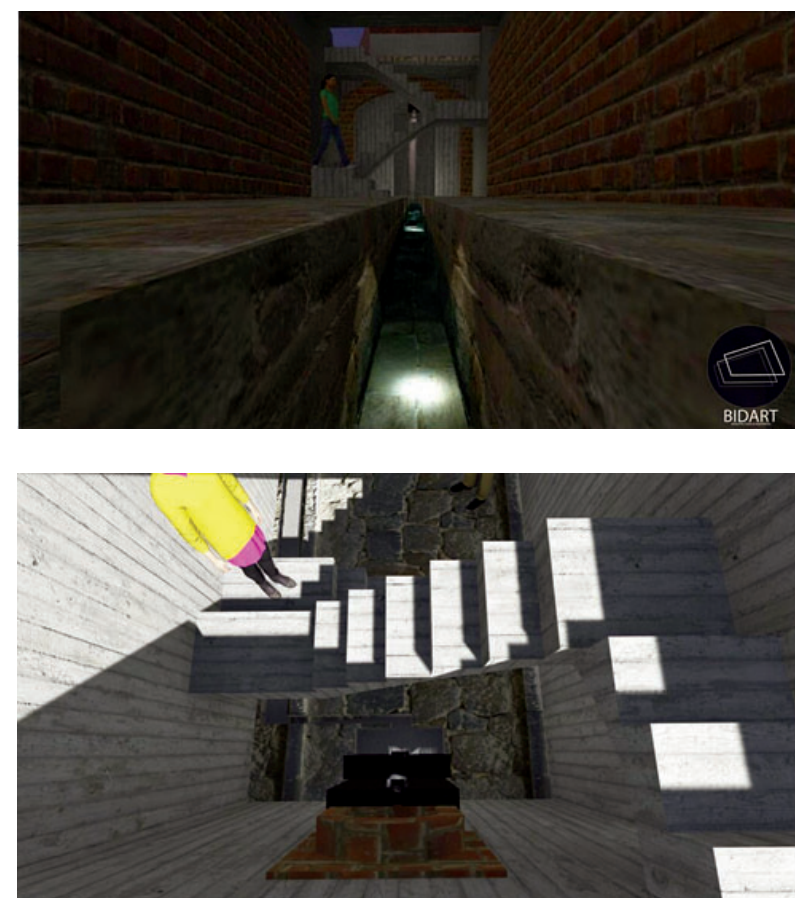

antiguo acueducto existente. Esto permitiría ofrecer a la comunidad y a sus visitantes una experiencia con el cuidado del ciclo del agua, el control de la erosión y la recreación en el borde costero.

De este modo, el proyecto consta de intervenciones en diversos puntos, destacando la cabecera cubierta por el Bosque Esclerófilo, el fondo de quebrada y el desagüe de sus cauces en el Puerto de Valparaíso.

Restauración del ecosistema de la quebrada costera mediante un pabellón verde abierto a la comunidad

Para restaurar el ciclo natural del agua cosechada por la cuenca, debe reconectarse el puerto con su parte alta mediante la reforestación nativa y la construcción de taludes de biofiltración, siendo piezas claves además para evitar la contaminación y el arrastre por la pendiente de sedimentos hacia el mar.

De esta forma, la decantación del flujo de agua genera pozones contenidos por taludes, que mediante filtros en base a plantas macrófitas y piscinas de decantación con filtros minerales, tales como arena, grava y bolones, logran la recuperación de las aguas y sus sedimentos. Esto da lugar a un paisaje capaz de depurar el agua, mejorando la calidad de vida de las comunidades que habitan e lugar y la posibilidad de reutilización de las aguas.

A su vez, es importante mencionar que la construcción del parque respeta la morfología de la cuenca y de las construcciones de Valparaíso, consolidando un espacio público donde las personas que viven en torno a la cuenca lo hagan en cercanía y con un mayor entendimiento del mundo natural.

\section{Acueducto y Bóveda de Agua}

El proyecto propone dar lugar a esta caída de agua de vertiente natural como el acceso a un museo in situ, el cual estaría ubicado en la bóveda que recorre subterráneamente el Barrio Puerto de la ciudad, poniendo en valor este sistema de ingeniería urbana histórico. Así también, tras acoger el flujo natural de agua que dio origen a la construcción de este espacio, se vuelve a dar vida a esta reliquia arqueológica.
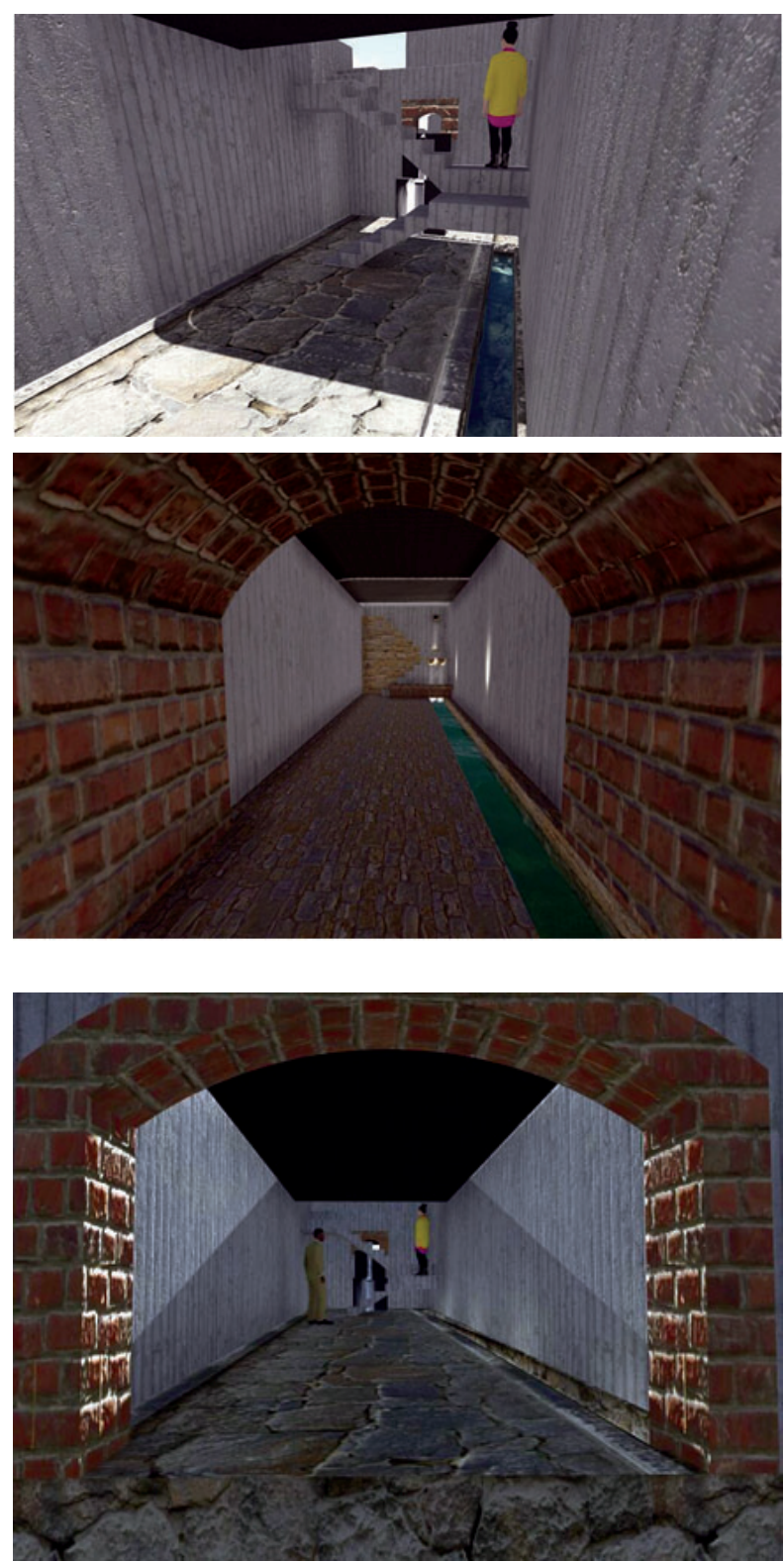

Figura 16. Maqueta 3D del recorrido por el museo in situ. Fuente: Bidart Arquitectos.

$>$ Figura 17. Maqueta 3D del acceso al acueducto. Fuente: Bidart Arquitectos.

$>$ Figura 18. Maqueta 3D del acceso al acueducto visto desde el interior. Fuente: Bidart Arquitectos.

$>$ Figura 19. Maqueta 3D del recorrido por la bóveda. Fuente: Bidart Arquitectos.

Figura 20. Maqueta 3D del recorrido por la bóveda. Fuente: Bidart Arquitectos. 


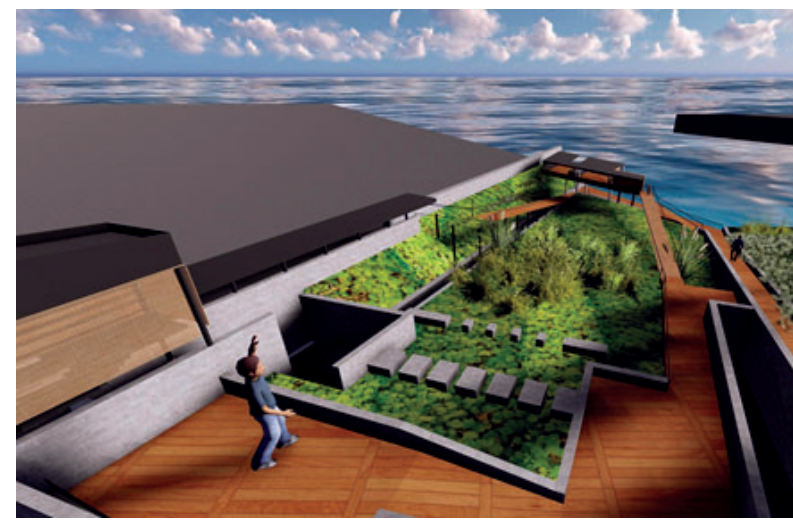

El museo está limitado geométricamente entre la bóveda anteriormente mencionada y el muro de contención que soporta las edificaciones colindantes mediante dos muros de hormigón de 9 $\mathrm{m}$ de largo que tienen una luz de 3,2 m entre ellos, la cual tiene la función de amarre perimetral de la estructura y soporte para las vigas maestras que resisten la losa de la calzada.

De esta forma, este espacio se convertiría en un proyecto piloto de transmisión y actualización de la información arqueológica, convirtiendo los diferentes estratos históricos en un soporte de espacio público.

\section{Hub del Agua, desembocadura del cauce al puerto de Valparaíso}

El término inglés hub puede traducirse al español como intercambiador, centro o punto de conexión, concentrador o nodo, siendo un anglicismo que se emplea actualmente para referirse a una especie de centro neurálgico en torno al cual se concentra y se mueve un determinado sector (Fundación del Español Urgente [FundéuRAE], 2019).

En este sentido, el Hub del agua, ubicado en pleno puerto de la ciudad, está ideado como un espacio de aprendizaje con un centro de decantación que regula el caudal del cauce San Francisco y que permite consolidar la desembocadura al borde mar en un paseo público que busca integrar diseño, paisajismo y conservación.

Este recorrido construye la contención del agua limpia, clave de cualquier sistema eco-sistémico, dando lugar a un espacio para la contemplación y aprendizaje sobre el ciclo natural del agua y su papel elemental para la continuidad de la vida en el planeta.

\section{BIBLIOGRAFÍA}

Alvarez, L. (2001). El origen de los espacios públicos en Valparaíso. Recuperado de https://web.uchile.cl/vignette/revistaurbanismo/n4/alvarez/alvarez.html

Daily, G. (1997). Introduction: What are ecosystem services? Recuperado de https://www.raincoast.org/library/wpcontent/uploads/2012/07/Daily_1997_Natures-serviceschapter-1.pdf

Fundación del Español Urgente. 07/06/2019. hub, alternativas en español. Recuperado de https://www.fundeu.es/recomendacion/hub-alternativas-en-espanol/

García, R (1996). Proyecto Corredor Biológico Mesoamericano. Recuperado de https://www.sica.int/documentos/informe-tecnico-regional-proyecto-corredor-biologico-mesoamericano_1_9301.html

Gurrutxaga, M. y Lozano, P. (2008). Evidencias sobre la Eficacia de los Corredores Ecológicos: ¿Solucionan la problemática de fragmentación de hábitats?. Recuperado de: https:// revistas.ucm.es/index.php/OBMD/article/view/OBMD0808110171A/21302

Martinez Duarte, J. (2006). Enfoque Sistémico en la Investigación de Cuencas Hidrográficas. Recuperado de https://www. redalyc. org/articulo.oa?id=357935464003

Muga, E. y Rivas, M. (2009). Mutaciones y Cambios en la Estructura Urbana del Área Metropolitana de Valparaíso. Recuperado de http://geografia.uc.cl/images/serie_GEOlibros/ del_pais_urbano/Eliana_Muga_Marcela_Rivas.pdf

Figura 21. Maqueta 3D del hub del agua con vista hacia el puerto. Fuente: Fundación Cuencas Chile y Bidart Arquitectos. 

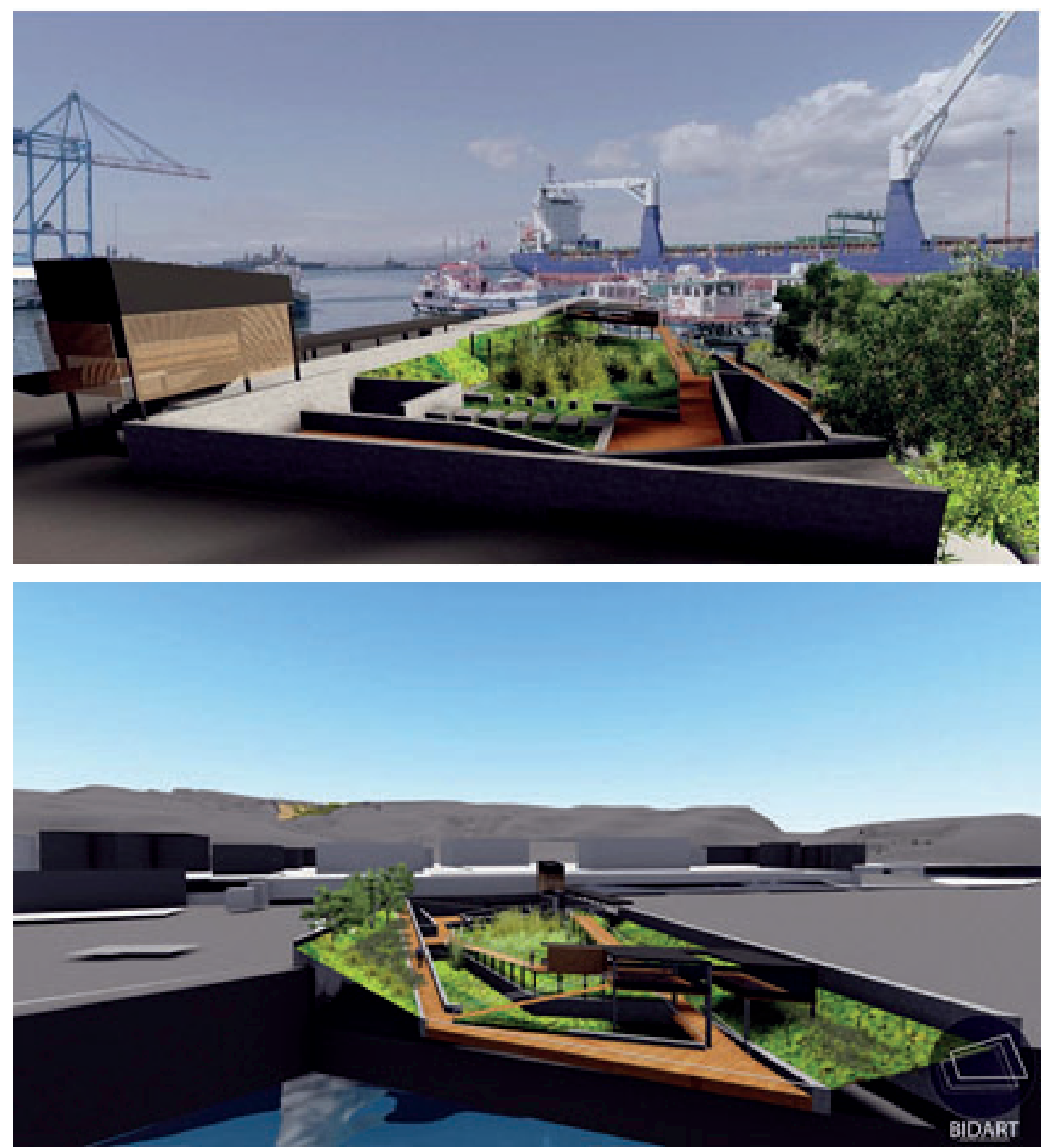

Urrutia, R. y Lanza, C. (1993). Catástrofes en Chile 1541-

1992. Recuperado de http://bosques.ciren.cl/hand-

le/123456789/18899

Urteaga, L. (1980). Miseria, Miasmas y Microbios. Las Topografías Médicas y el Estudio del Medio Ambiente en el siglo XIX. Recuperado de http://www.ub.edu/geocrit/geo29. htm

$>$ Figura 22. Maqueta 3D del hub del agua. Fuente: Fundación Cuencas Chile y Bidart Arquitectos.

> Figura 23: Maqueta 3D del hub del agua visto desde el mar. Al fondo en color se visualiza la naciente de la quebrada. Fuente: Fundación Cuencas Chile y Bidart Arquitectos. 